\title{
Identification automatique des diatomées de la Merja fouarate : Une alternative à la détermination et à la classification manuelle
}

\author{
Nouzha CHAHBOUNE, Mohamed MEHDI et Allal DOUIRA \\ Université Ibn Tofail, Faculté des Sciences, \\ Laboratoire de Botanique et de Protection des Plantes, B. P. 133, Kénitra, Maroc. \\ *Auteur correspondant e-mail - : chahbounenouzha@yahoo.fr ; douiraallal@hotmail.com ; mehdimina@hotmail.com \\ Original submitted in on $8^{\text {th }}$ May 2015. Published online at www.m.elewa.org on $30^{\text {th }}$ September 2015 \\ http://dx.doi.org/10.4314/jab.v93i1.2
}

\section{RESUME}

Objectif : L'objectif de ce travail consiste à développer une méthode automatique d'analyse de formes et de classification des Diatomées par le traitement d'images numérisées

Méthodologie et résultats : L'identification automatique des diatomées a été effectuée via l'utilisation d'un logiciel Image $\mathrm{J}$. Les résultats obtenus se sont montrés très satisfaisants, donnant des taux de bonne classification supérieurs à $96 \%$ et surpassant même l'identification manuelle. Ce travail s'appuie sur les méthodes de squelettisation des diatomées et de l'utilisation du code de Freeman pour codifier le contour, ensuite sur l'extraction des points d'intérêts des ornementations afin de ne préserver qu'un sous ensemble restreint et pertinent de pixels et finalement sur l'indexation croisée entre les diatomées et les ornementations afin d'accélérer le processus d'identification.

Conclusion et application de la recherche : Les particularités de certaines espèces potentiellement plus complexes n'ont pu être résolues et l'élaboration d'un programme informatique qui automatise l'identification des Diatomées, qu'elle que soit leur spécificité, reste encore difficile à résoudre et ne pouvait de ce fait être généralisable. Cependant les recherches continuent et s'appuient fortement sur les retombées de l'Intelligence artificielle.

Mots clés : Diatomées, actuel, identification automatique, traitement numérique de l'image, morphologie mathématique, Fouarate, Kenitra, Maroc.

Automatic identification of Fouarate Merja diatoms: An alternative to manual determination and classification

\section{ABSTRACT}

Objective: The objective of this work consists of developing an automatic method of determination and classification of diatoms by processing scanned images.

Methodology and Results : Automatic identification of diatoms was performed via the use of a software ImageJ. The results obtained were shown very satisfactory, giving accurate classification superior to $96 \%$, even exceeding the manual identification. This work is based on diatoms squelettization methods, and the 

fouarate : Une alternative à la détermination et à la classification manuelle

use of the Freeman code to codify contour, then on the extraction of the ornamentations points of interests in order to only preserve restricted and relevant subset of pixels, and finally on the cross-indexing between the diatoms and the ornamentations aiming to accelerate the identification process.

Conclusion and application of research: The special features of some potentially more complex species were resolved and the development of a computer program that automates the identification of diatoms, that of their specificity, is still difficult to solve and could therefore be generalized. But research continues and relies heavily on the impact of Artificial Intelligence.

Keyword: Diatoms, Automatic Identification, actual Image Processing, Mathematical Morphology, Fouarate, kenitra, Maroc.

\section{INTRODUCTION}

On présente, dans cette étude, une alternative aux techniques manuelles de détermination des diatomées. La problématique de l'identification et de la classification des diatomées s'avère délicate, même pour les spécialistes. Le nombre d'espèces connues est très grand (11000 environ) et l'identification à l'œil nu est une source d'erreur (Kelly et al., 2002). Ces difficultés entraînent inévitablement un processus d'analyse long,

\section{MATERIEL ET METHODES}

Située dans le plateau de la Mamora (Thauvin, 1966), entre la plaine argileuse plio-quaternaire du Rharb et la Meseta occidentale schisto-gréseuse du Paléozoique (Michard, 1976), la Merja Fouarat est le siège d'une

fastidieux et peu sûr (Guo, 2004 ; Loke, 2002). Face à ce problème, cette étude propose, la création d'un processus automatisé, fondé sur les techniques utilisées dans la reconnaissance des formes des diatomées afin de les identifier et de les classer automatiquement. Pour cela, une comparaison entre une identification manuelle et une identification automatique sera effectuée, afin de montrer l'efficacité de cette méthode.

nappe phréatique située dans les sables et gréscalcaires du Plio-Villafranchien (Combe, 1975) à climat méditerranéen et sous influence océanique prononcée (Subhumide en étiage, tempérée en hiver).

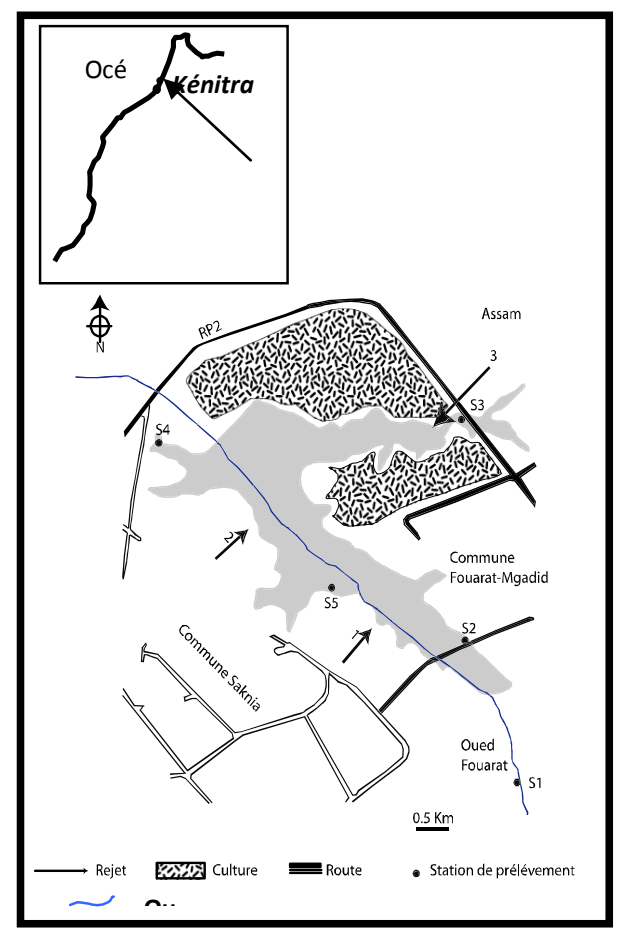

Figure 1 : Carte de la Merja Fouarat 


\section{Chahboune et al. J. Appl. Biosci. 2015 Identification automatique des diatomées de la Merja fouarate : Une alternative à la détermination et à la classification manuelle}

Échantillonnage : Au niveau des cinq stations situées tout au long de la Merja Fouarate, quatre campagnes de prélèvements de diatomées ont été effectuées, en vue de dresser un inventaire des espèces diatomiques de la Merja Fouarate. Les diatomées sont collectées sur des galets grâce à des brosses à dents notamment en dehors de toute période de crue.

Préparation du matériel collecté: Les échantillons subissent tout d'abord une digestion dans du peroxyde d'hydrogène porté à ébullition, afin de détruire toute matière organique présente dans l'échantillon. Quelques gouttes d'acide chlorhydrique peuvent être ajoutées pour éliminer les résidus de carbonates de calcium. Ensuite l'échantillon est centrifugé à $4500 \mathrm{tr} / \mathrm{min}$. Puis le culot formé est récupéré et dilué dans l'eau distillée, à plusieurs reprises (rinçage).

Préparation des lames microscopiques: Suite à l'échantillonnage on procède à la préparation des lames microscopiques, selon les méthodes françaises de bioindication standardisés et définis dans la norme NFT 90354 (Afnor, 2007). La suspension contenant les diatomées doit être montée entre lame et lamelle puis l'analysée au microscope.

Identification microscopique des Diatomées: Pour l'identification des Diatomées au microscope, nous avons fait appel à la nomenclature de Germain (1981), Krammer \& Langbertalot (1986, 1988, 1991, 1991, 2000). Les taxons sont identifiés, grâce à l'observation des frustules au microscope optique à un grossissement 1000 fois. La détermination est effectuée en se basant sur l'analyse des éléments morphologiques suivants (Lavoie et al., 2008), à savoir :

- La forme générale des frustules, la forme de l'aire centrale, la forme des stries,

- La densité des stries, le type et la taille des ponctuations, la présence de stigma,

- La présence, la forme et la densité des fibules et des côtes, La présence d'épines,

- La forme de l'apex, la présence ou l'absence du raphé, la position du raphé et

- La forme générale du raphé (Fig 2).

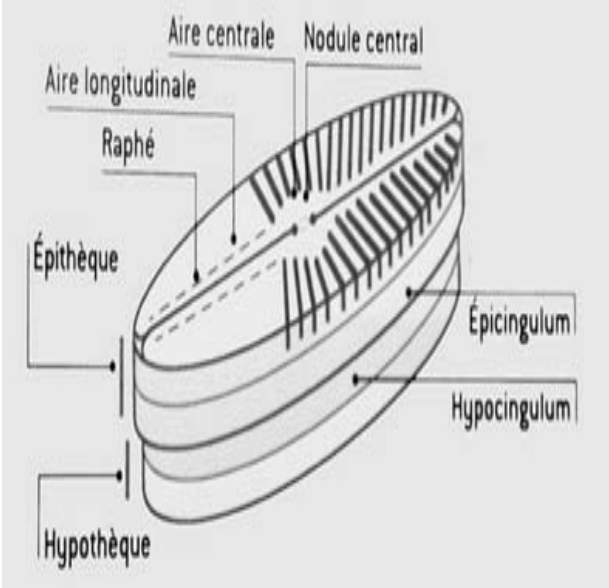

Figure 2 : Structure d'un frustule de diatomée pennale (Naviculaceae

L'identification des Diatomées en lames minces, minimum 400 valves, nous permet d'obtenir des richesses spécifiques et des abondances relatives stables (Prygiel et al., 2002). II est important de procéder au balayage de la lamelle, afin d'observer les diatomées de façon aléatoire et d'éviter de compter doublement le même spécimen.

Identification automatique des diatomées: L'identification automatique et la classification des
Diatomées reposent sur des techniques couramment utilisées pour la reconnaissance des motifs dans une image. (Du Buf H. et al., 1999). L'ensemble de techniques passe par quatre étapes :

- Étape 1: phase de prétraitement: cette étape consiste à atténuer le bruit et à rehausser les contrastes afin de ressortir les motifs à analyser ;

- Étape 2 : phase de segmentation des différentes régions homogènes de l'image et l'identification des 


\section{Chahboune et al. J. Appl. Biosci. 2015 Identification automatique des diatomées de la Merja fouarate : Une alternative à la détermination et à la classification manuelle}

motifs de la structure interne des Diatomées ;

- Étape 3: phase d'analyse et d'extraction des caractéristiques des motifs choisis.

- Étape 4 : phase d'identification et de classification des différentes espèces.

Ce travail d'identification automatique des diatomées s'appuie sur le logiciel Imagej. Une application de traitement numérique d'images développée à la National Institute of Health (Wayne, 1997). ImageJ est un puissant logiciel de traitement et d'analyse d'images scientifiques, autant par ses fonctions intrinsèques que par le riche ensemble de fonctions délivrées sous forme de plugins. De plus, il est dans le domaine public. Ce logiciel s'utilise dans tous les domaines scientifiques et demeure l'outil privilégié pour l'analyse d'images en sciences du vivant.

Élaboration d'une base d'images de diatomées: Avant toute identification, il est nécessaire d'élaborer une base d'images. II s'agit de 10 espèces de Diatomées de la Merja Fouarate. II ne s'agit nullement d'un échantillon représentatif, notre but ici est de vérifier l'efficacité de notre méthode d'identification. Ultérieurement, il serait possible d'alimenter la base d'images Diatomiques. L'organisation de la base est fondée sur une indexation croisée. Le principe est le suivant: les attributs représentant chaque Diatomée sont sauvegardés dans un vecteur. Chaque attribut du vecteur est indexé aux Diatomées qui le possèdent et chaque Diatomée est indexée aux attributs qui lui appartiennent. Durant l'identification, Iorsqu'un attribut est sélectionné, l'analyse continue à partir des Diatomées liées à cet item, ainsi en deux ou trois itérations d'être face à 1 ou deux candidats.

Méthode de numérisation de diatomées: Le traitement numérique des images, fondé sur la morphologie mathématique (Serra 1988), met en correspondance une image que l'on cherche à identifier avec une image se trouvant dans la base d'images (Bres S., et al. 2003). Pour permettre cette recherche de similitude, le système extrait les caractéristiques de la Diatomée à analyser. Ces opérations consistent à produire sous forme matricielle le contour et les ornementations. Une fois ces opérations effectuées, il cherche à trouver, la Diatomée correspondante, selon les critères que nous avons développés ci-dessus, à savoir la pertinence des items et la base de connaissances. 


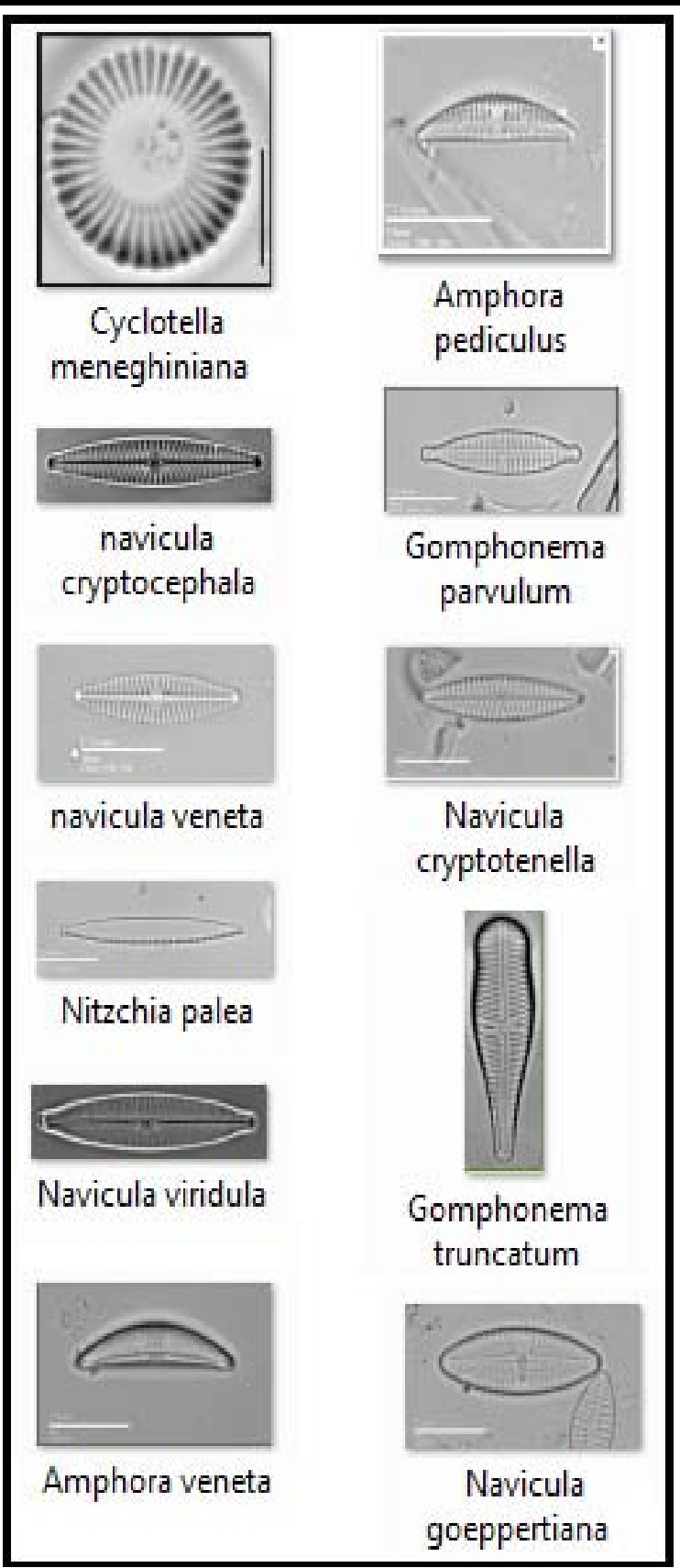

Figure 3 : Exemple des diatomées de la Merja fouarate

Extraction du contour: La classification de Diatomées que nous effectuons est fondée sur le contour (Deriche, 2004). En effet, cette caractéristique permet d'organiser la base d'images diatomiques en fonction des espèces. Cette opération permet de réduire le nombre de candidats à analyser de manière considérable. Pour se faire, nous procédons de la manière suivante : Process/Binary/Make Binary. 
Chahboune et al. J. Appl. Biosci. 2015 Identification automatique des diatomées de la Merja fouarate : Une alternative à la détermination et à la classification manuelle

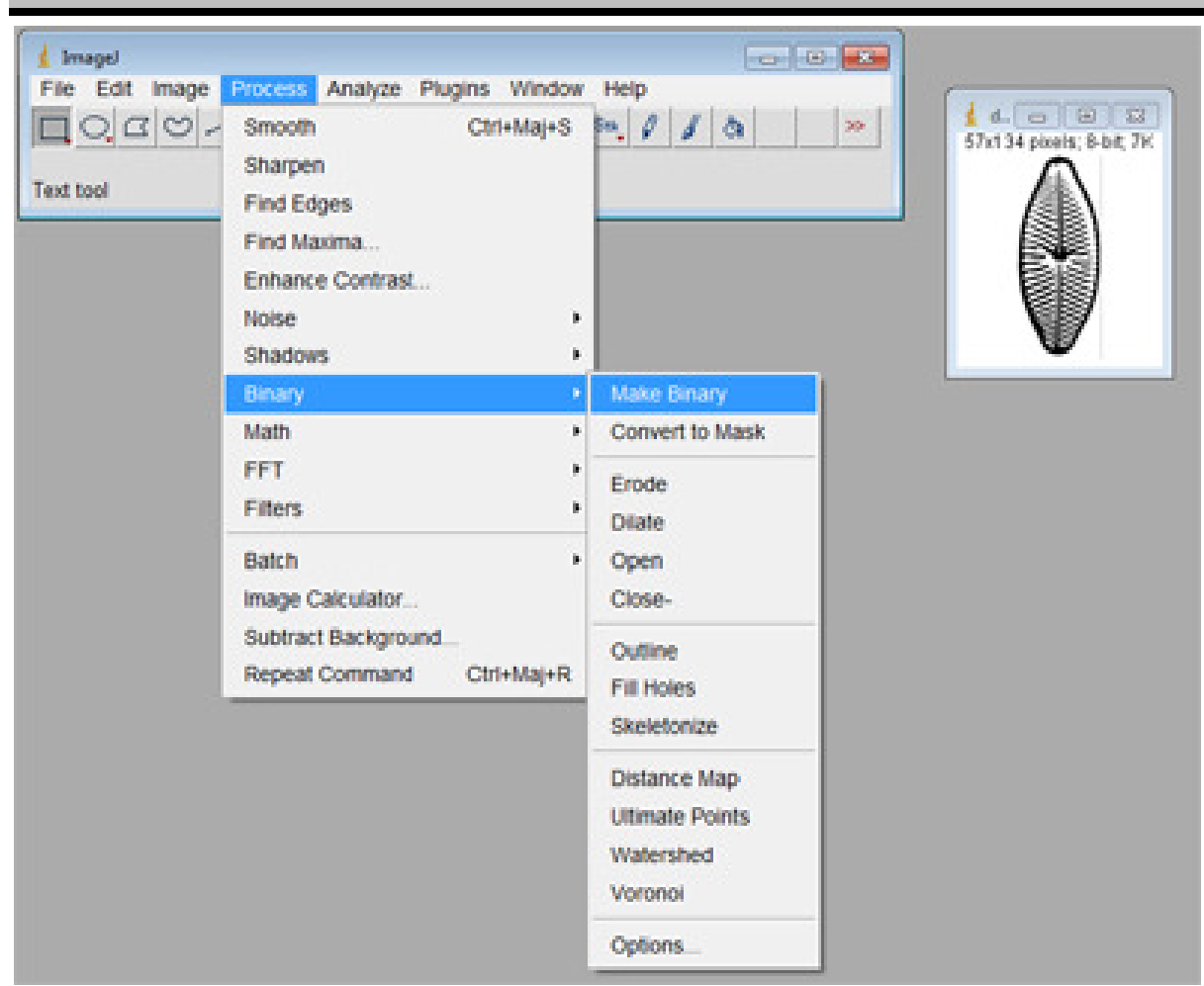

Figure 4 : Binarisation de la diatomée

Ensuite, nous utilisons le filtre de remplissage qui noircit l'intérieur, ce que l'on peut réaliser grâce à la fonction Process/Binary/Fill Holes.

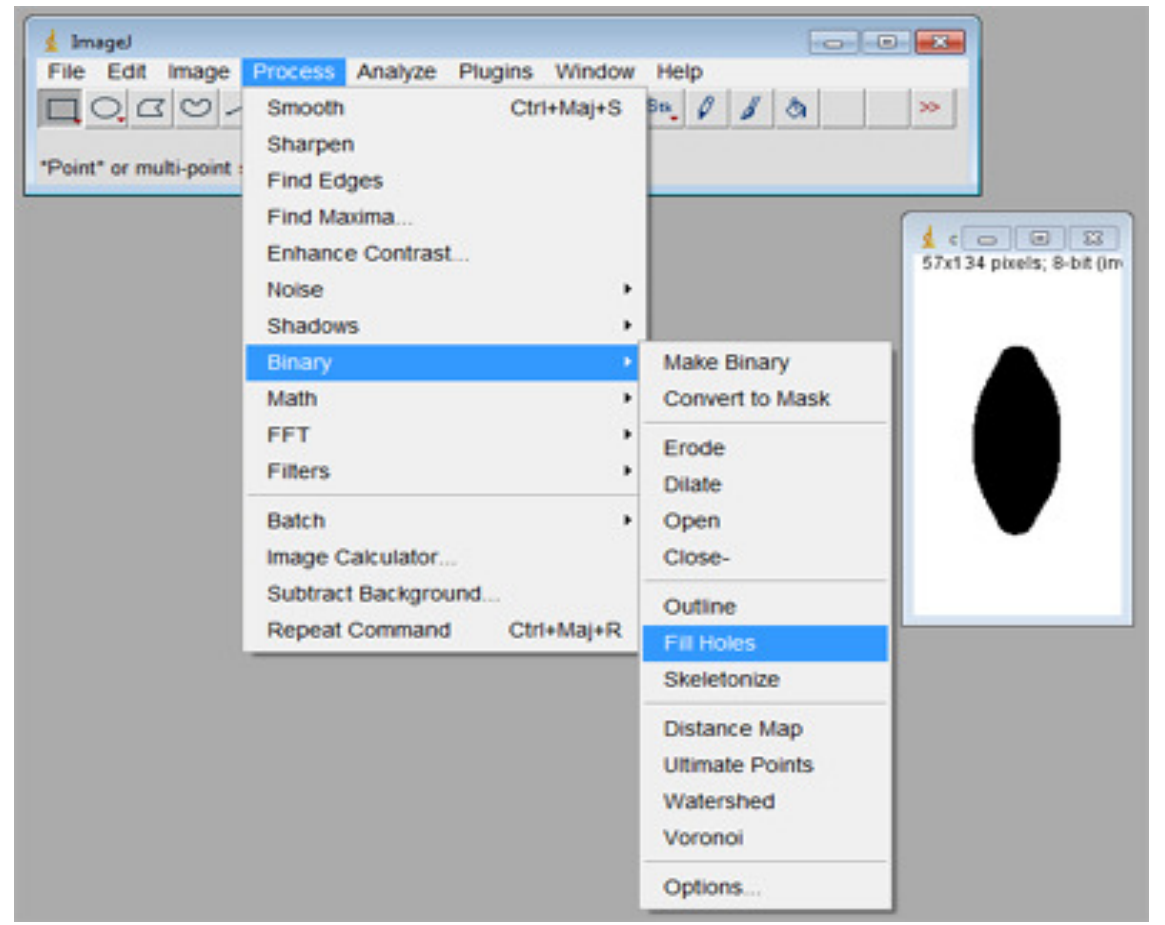

Figure 5 : Fill Holes de l'image de la diatomée 
Chahboune et al. J. Appl. Biosci. 2015 Identification automatique des diatomées de la Merja fouarate : Une alternative à la détermination et à la classification manuelle

Le troisième filtre se fonde sur le filtre de Sobel qui permet d'obtenir le contour. La fonction qui utilise ce filtre est Process/Find Edges

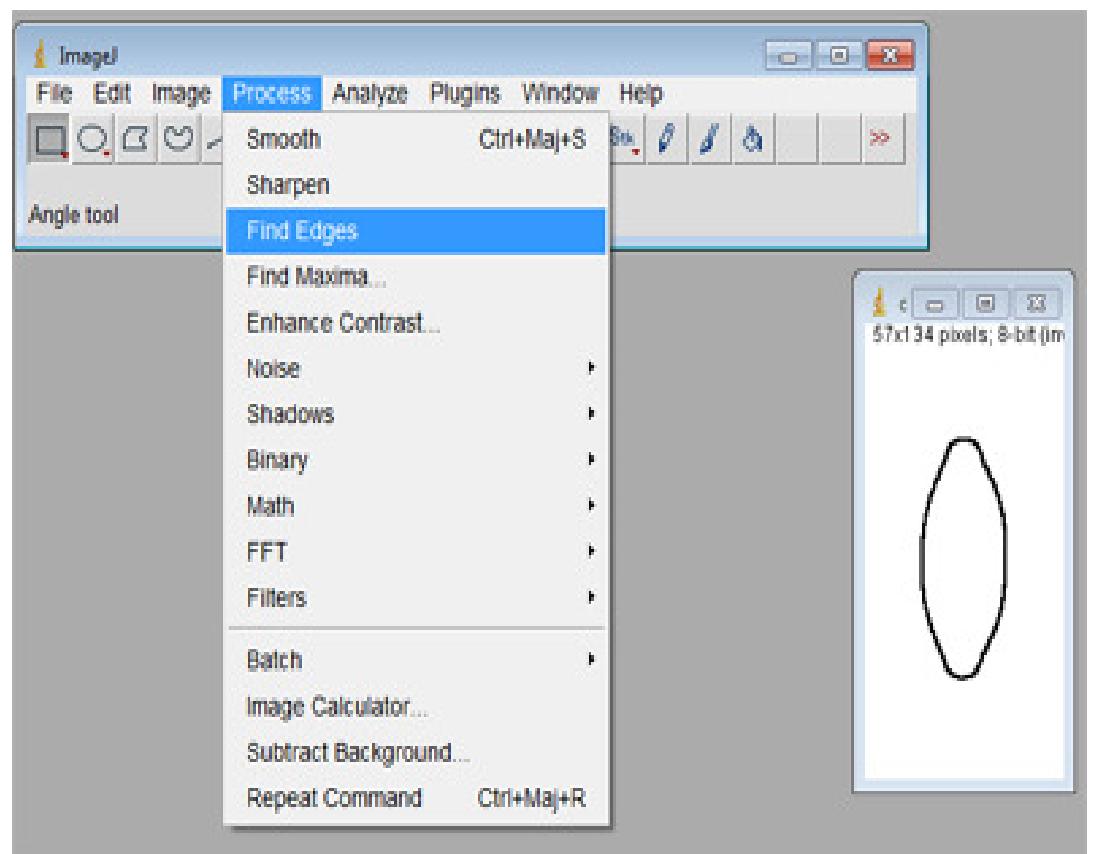

Figure 6 : Extraction du contour de la diatomée

Le quatrième filtre correspond à la squelettisation du contour. $\underline{\text { Process/Binary/Skeletonize }}$

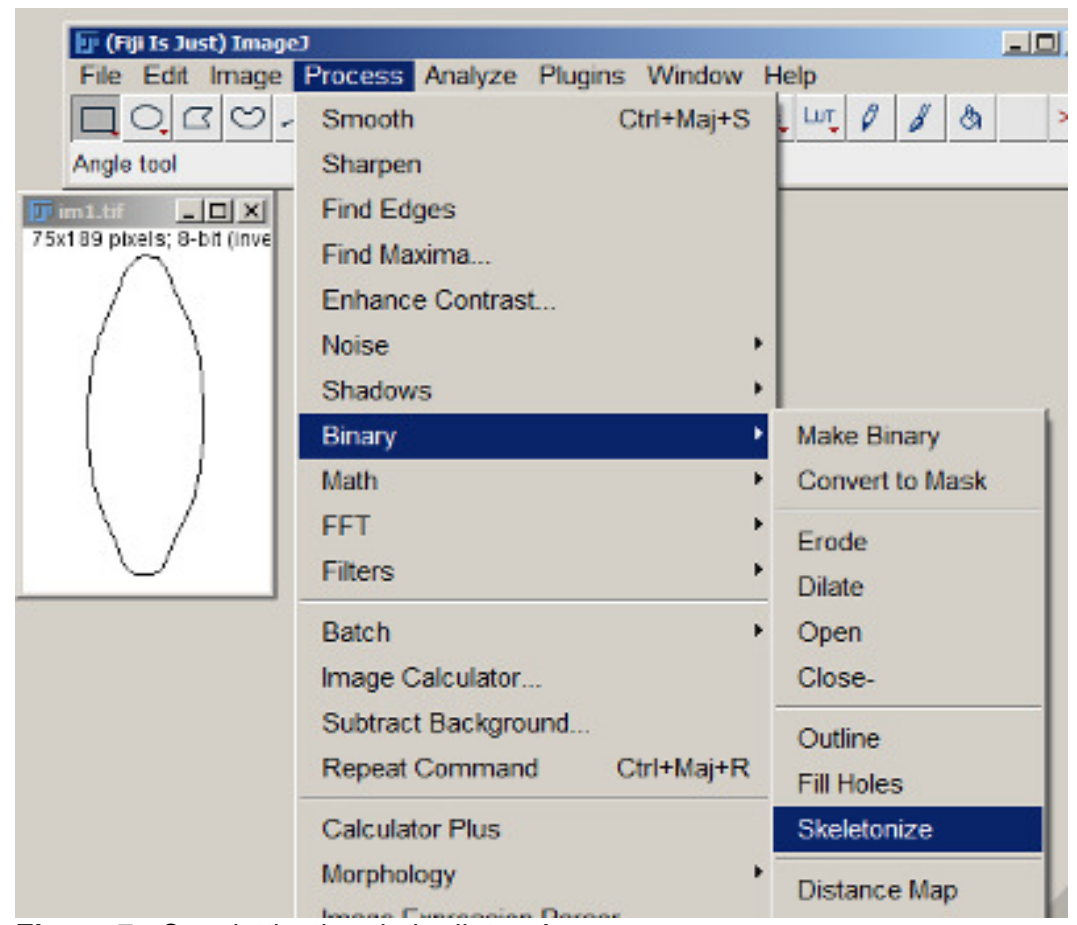

Figure 7 : Squelettisation de la diatomée 
Chahboune et al. J. Appl. Biosci. 2015 Identification automatique des diatomées de la Merja fouarate : Une alternative à la détermination et à la classification manuelle

La cinquième opération consiste à obtenir le vecteur correspondant en comptant le nombre de chiffres pairs et le nombre de chiffres impairs, selon la formule suivante :

$(741 \times 1)+(151 \times \sqrt{ } 2) \approx 987.55$

Extraction des ornementations: Afin de récupérer les stries des zones de motifs, nous commençons par effectuer un seuillage afin d'obtenir une image binarisée. L'image doit être en grayscale (Dubuisson 1990). Le seuillage, analyse chaque pixel de la matrice représentant l'image. Si la valeur de ce pixel est inférieure à un seuil, par exemple 128, on remplace la valeur de ce pixel par 0 , sinon on la remplace par 255 . C'est ce qui nous permet d'obtenir une image binarisée.
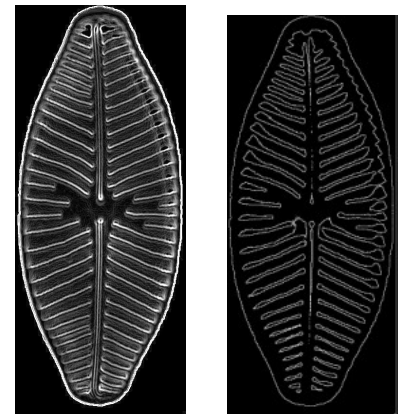

Figure 8 : Binarisation de l'image de la diatomée

Une fois cette étape achevée, nous utilisons une opération morphologique qui effectue une soustraction entre deux matrices. Dans notre cas, cela concerne l'image que nous venons de squelettiser avec l'image contour.

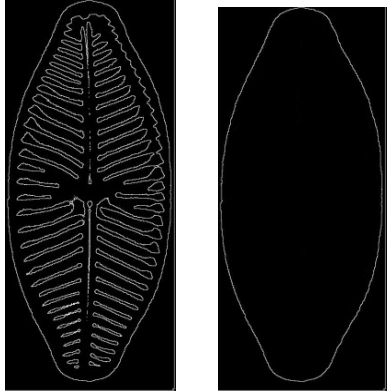

Figure 9 : Détermination de contour de la diatomée

Ce qui nous permet d'obtenir le frustule sans le contour.

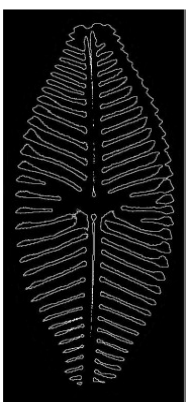

Figure 10 : Détermination du fond de la diatomée

Cette opération s'effectue en utilisant la commande Image Calculator à partir du Menu Process, qui calcule la différence entre une image binarisée et sa dérivée dilatée et propose le résultat dans une troisième image.

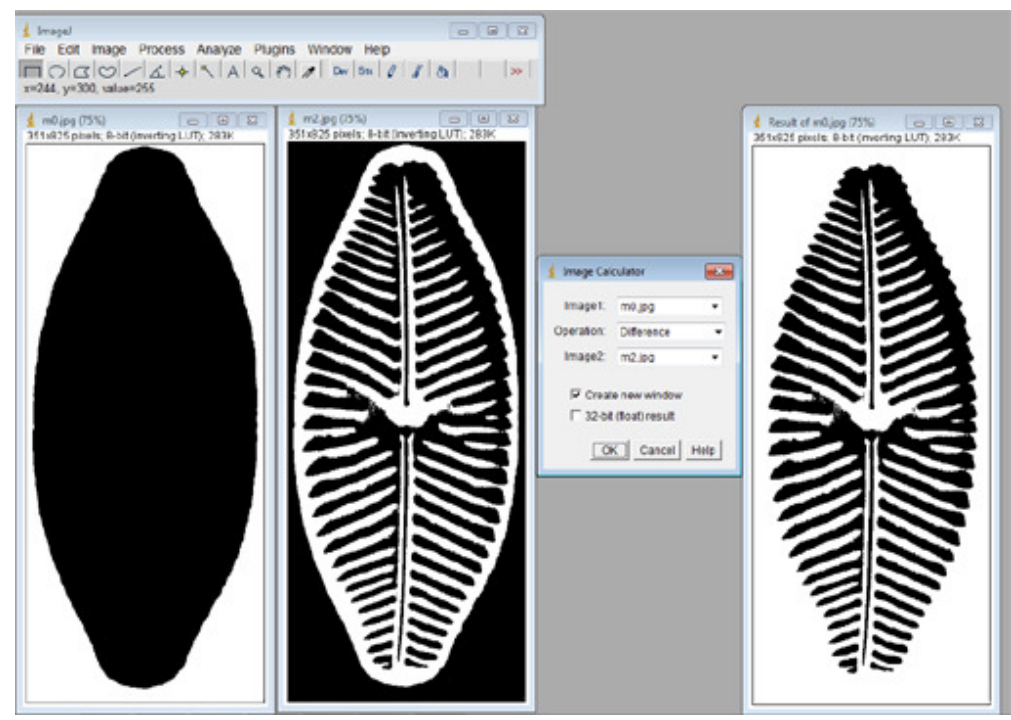

Figure 11 : Extraction d'ornementation de la diatomée 
METHODOLOGIE ET RESULTATS :

Identification diatomique par microscope: $A u$ niveau de la Merja Fouarat et au cours des 4 saisons (hiver, printemps, été et automne) l'inventaire et l'abondance relative des espèces de Diatomées dans les cinq stations prospectées, montrent que les prélèvements effectués ont fourni une centaine d'espèces environ. Ces Diatomées étaient groupées en 21 genres, avec une erreur de $4 \%$ car plusieurs organismes appartenant aux genres Navicula, Nitzschia, Gomphonema et Amphora n'ont pas pu être identifiés au niveau de l'espèce, comme cela a été développé par (Ébang Menye et al. 2012).

Tableau 1: Les différents genres identifiés au niveau de la Merja Fouarate

\begin{tabular}{|l|}
\hline Genre de diatomées \\
\hline Achnanthes \\
\hline Amphora \\
\hline Anomoeoneis \\
\hline Caloneis \\
\hline Cocconeis \\
\hline Cyclotella \\
\hline cymbella \\
\hline Diploneis \\
\hline Epithemia \\
\hline Eunotia \\
\hline Fragilaria \\
\hline Gomphonema \\
\hline Gyrosigma \\
\hline Navicula \\
\hline Nitzschia \\
\hline Pinnularia \\
\hline Pleurosira \\
\hline Rhopalodia \\
\hline Thalassiosira \\
\hline
\end{tabular}

Les résultats de nos comptages montrent l'abondance de quelques espèces durant toute l'année et dans toutes les stations, il s'agit notamment des espèces résistantes et très résistantes à la pollution organique : Cyclotella meneghiniana Kützing, Navicula veneta Kützing, Gomphonema parvulum Kützing et Navicula subminuscula Manguin. Le type d'eau potentielle au niveau de la Merja et Oued Fouarat est eutrophe. Cette situation est confirmée par la présence des espèces sensibles et très sensibles telle que Navicula cryptotenella Lange-Bertalot, Cymbella sinuata Gregory, Amphora pediculus (Kützing) Grunow et
Cocconeis placentula var. euglypta (Ehrenberg) Grunow. Dans les stations polluées (3, 4 et 5$)$, les espèces sensibles à la pollution organique disparaissent au profit des espèces résistantes, tout en gardant le caractère eutrophe: Navicula veneta Kützing, Navicula subminuscula Manguin, Cyclotella meneghiniana Kützing et Amphora veneta Kützing. Lors de l'identification au microscope, une attention spéciale a été consacrée aux taxons considérés de façon générale comme difficiles à déterminer et pouvaient être confondue avec d'autre forme. II s'agit essentiellement des taxons et groupes de formes suivants (Hurlimann, 2007) :

- Amphora pediculus et Amphora. Inariensis. Ces deux taxons morphologiquement proches devraient être différenciés pour des raisons écologiques.: Amphora pediculus, avec une valeur indicatrice $D$ de 5.0 , se rencontrent dans des eaux nettement polluées, $A$. inariensis, avec $D=3.5$, préfère plutôt des eaux très peu ou faiblement polluées.

- Gomphonema parvulum se présente sous trois variétés : parvulum, exilissimum et parvulum $f$. saprophilum. Les deux variétés parvulum et parvulum $f$. saprophilum présentent du point de vue écologique la même valeur $D$, il devrait s'agir très vraisemblablement de la même forme. Mais il est important de distinguer Gomphonema parvulum var. parvulum, rencontrée dans les eaux fortement polluées, de Gomphonema parvulum var.exilissimum (nouvelle dénomination : Gomphonema exilissimum Lange-Bertalot et Reichardt) qui, avec une valeur $D$ de 3.0 , se rencontre dans les eaux très faiblement polluées.

- $\quad$ La ressemblance morphologique autour de Navicula cryptotenella, $N$ menisculus, $N$. reichhardtiana, $N$. aquaedurae et $N$. cryptotenelloides est très grande, et il est difficile de les distinguer (Lange-Bertalot, 2001). Tous les taxons mentionnés ont une valeur $D$ de 4.0 ; ils ne se différencient donc écologiquement qu'au niveau du groupe et non pas des différents taxons.

- Nitzschia fonticola et N. tropica: N. tropica a été répertoriée en Afrique centrale et sa présence a été signalée récemment dans le cours supérieur de la Loire (Prygiel \& Coste, 2000). Cette espèce est restée ignorée jusqu'à ce jour en raison de confusions taxinomiques, elle peut être confondue avec les formes volumineuses de $N$. fonticola et autre espèces de Nitzschia morphologiquement proche exemple de $\mathrm{N}$. costei et N. fossilis (Office de l'eau Réunion, 2009).

- Gomphonema gracile et Gomphonema affine, 


\section{Chahboune et al. J. Appl. Biosci. 2015 Identification automatique des diatomées de la Merja fouarate : Une alternative à la détermination et à la classification manuelle}

ces deux taxons morphologiquement très proches, peuvent être confondue lors de l'identification. Ces taxons en raison de leur appariement morphologiquement proches, présentent des confusions taxonomiques. Les erreurs de détermination sont donc proportionnelles à la similitude entre taxons : plus ils sont morphologiquement similaires, plus il devient difficile de les distinguer avec certitude. Ici, l'expérience du diatomiste dans l'art d'identification est fondamentale. Ce savoir-faire est ardu à acquérir et surtout à transmettre. C'est de cette difficulté que l'automatisation de l'identification des Diatomées, nous a semblé une piste à explorer.

Identification automatique des diatomées: En comparant deux Diatomées similaires, nous allons montrer comment l'identification automatique pourra remédier au problème précité. Nous avons vu, matériel et méthodes, que les Diatomées sont représentées dans la base d'images par leur forme et leurs ornementations sous la forme d'un vecteur. Ce sont les deux critères sur lesquels nous allons nous fonder pour effectuer des comparaisons et démontrer la puissance de l'identification automatique par rapport à une identification manuelle. II est à noter que chaque vecteur contient le nom de la Diatomée, son chain code (Freeman, 1961) déterminant le contour et la liste de points correspondant aux points d'intérêts.

Première étape: Supposons que nous voulons identifier la Diatomée suivante :

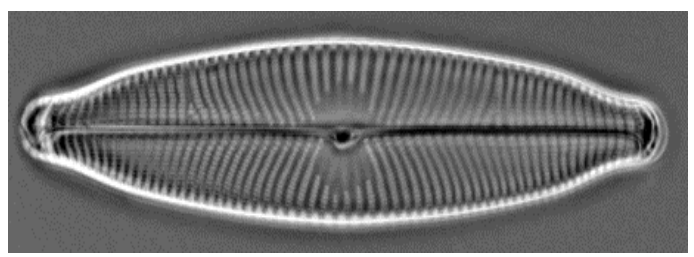

Figure 12 : Diatomée cible

Une macro ImageJ analyse l'image, ce qui nous permet d'obtenir les caractéristiques suivantes :

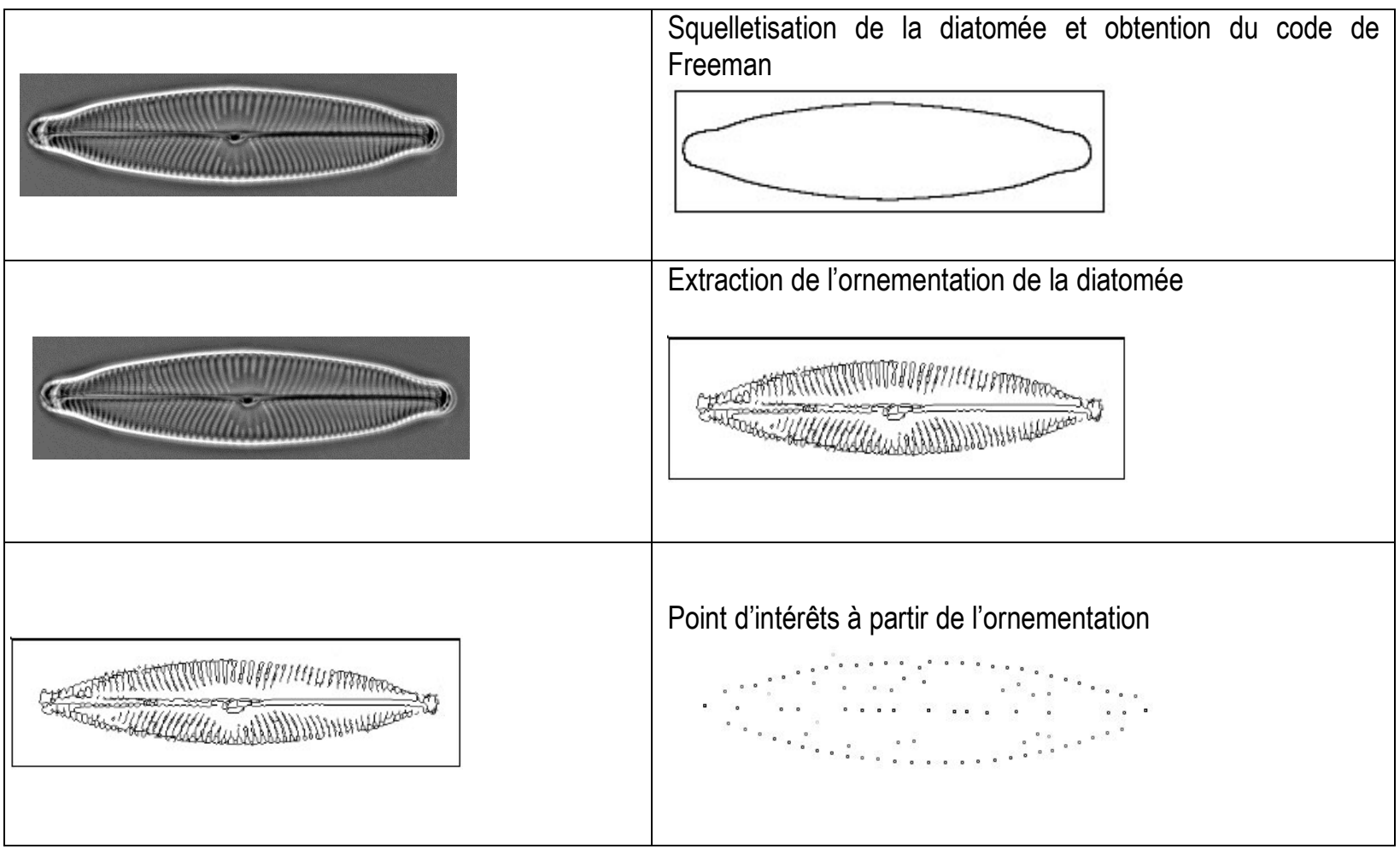

Figure 13 : Extraction des caractéristiques

Deuxième étape : La première caractéristique, permet d'obtenir le code de Freeman. Sachant que pour chaque contour nous obtenons son code, nous comparons dans ce cas seulement les codes entre eux. 
Chahboune et al. J. Appl. Biosci. 2015 Identification automatique des diatomées de la Merja fouarate : Une alternative à la détermination et à la classification manuelle

\begin{tabular}{|l|l|l|}
\hline $\begin{array}{l}\text { Une comparaison approximative à partir du } \\
\text { code nous donne selon notre exemple le } \\
\text { résultat suivant :Type de diatomées }\end{array}$ & Contour & Code de Freeman \\
\hline Amphora pediculus & & 831.93 \\
\hline Amphora veneta & & 955.29 \\
\hline Cyclotella meneghiniana & & 1199.24 \\
\hline Cymbella subequalis & & 997.49 \\
\hline Gomphonema parvulum & & 953.1 \\
\hline Gomphonema parvulum & & 987.55 \\
\hline Navicula cryptotenella & & 927.71 \\
\hline
\end{tabular}

Figure 14 : Résultats des codes de Freeman

Troisième étape : La deuxième caractéristique permet de comparer les ornementations des images candidates avec l'image cible, pour cela nous effectuons une comparaison à partir des points d'intérêts. Nous commençons par réduire l'image, nous choisissons la taille de $32 / 32$, ce qui permet de compresser l'image sans pour autant perdre de l'information. Ces points d'intérêts sont représentés sous la forme d'un vecteur.

Vect $=[3224,12824,13342,2975,11169,2082$, $5155,10531,13731,4004,1701,11941,14501,3622$, $11686,12583,6440,9256,7338,8618,13994,1708$, $8621,7086,11694,3119,3631,12080,13105,5172$, $6836,9140,14004,6069,11958,10423,13239,3512$, 7992, 3001, 4409, 2490, 3132, 5567, 12095, 7488, $3009,4033,10178,12355]$.

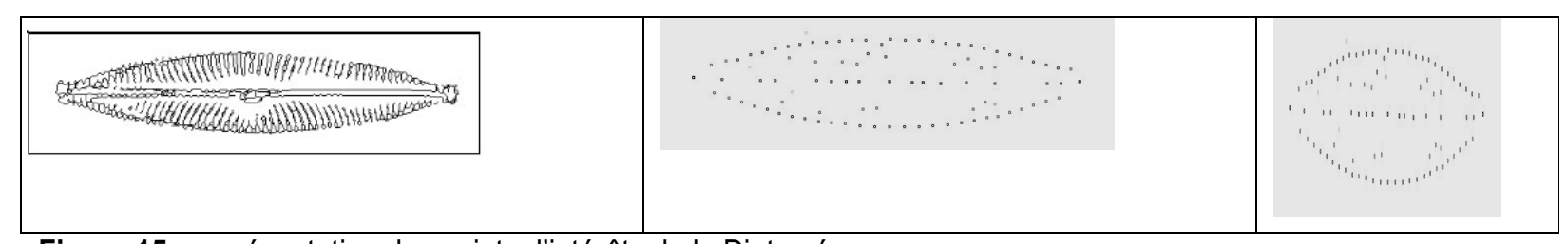

Figure 15 : représentation des points d'intérêts de la Diatomée

Ainsi, le contour permet de sélectionner un ensemble de diatomées, ensuite nous comparons par une indexation croisée à l'intérieur de cet ensemble les vecteurs correspondant aux points d'intérêt. 

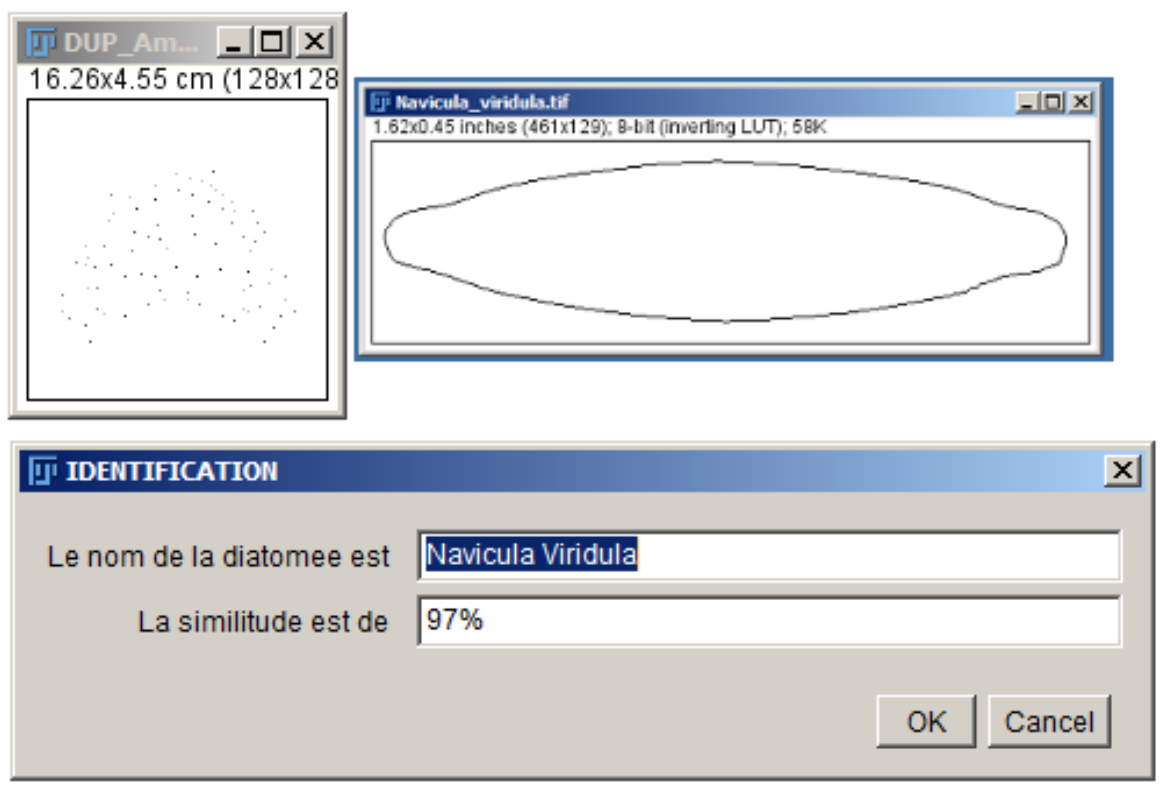

Figure 16 : Résultat de l'identification automatique de la diatomée

Dans notre exemple, le dernier candidat restant est «Navicula Viridula» qui correspond à notre image cible.

\section{CONCLUSION ET APPLICATION DE LA RECHERCHE :}

Le monde des Diatomées est diversifié en plusieurs groupes, genres et espèces. II présente beaucoup de variations morphologiques qui sont des éléments de base quant à leur détermination et leur classification. La reconnaissance automatique d'image apporte une aide précieuse à la fois pour la détermination des diatomées, et surtout comme outil pédagogique d'aide à l'identification au service de nouveaux diatomistes. Notre étude a visé à automatiser le processus d'identification des diatomées en s'appuyant sur des techniques d'analyse et d'interprétation d'images. En effet, la comparaison d'images suppose une image candidate, celle qu'il faut identifier et une base d'images dans laquelle il faut trouver l'image correspondante, permettant l'identification. Comparer des images pose des problèmes. Le premier est le choix de la résolution de comparaison. En effet, l'analyse d'images brutes est coûteuse en temps de calcul. II est de ce fait, intéressant de ne comparer que ce qui est nécessaire. L'extraction de descripteurs visuels sur l'image entière permet de réduire le nombre de calculs nécessaires. C'est ainsi que nous n'avons pas pris en considération tous les pixels des attributs à analyser, mais nous en avons prélevé un sousensemble représentatif, en s'appuyant sur l'approche des « point d'intérêts ». Le second problème concerne la méthode de comparaison, cette phase est particulièrement ardue, car la moindre différence entre les deux ensembles de caractéristiques peut faire échouer l'identification. Afin d'éviter ce problème, nous avons préconisé d'utiliser une méthode de comparaison basée sur la similitude approximative. L'utilisation d'un coefficient de similitude nous a permis d'avoir un peu plus de souplesse par rapport à une comparaison binaire trop discriminante. Pour l'élaboration de notre programme, nous nous sommes appuyés sur ImageJ, une application de traitement numérique d'images développée à la National Institute of Health. Au terme de ce projet, les résultats obtenus se sont montrés très satisfaisants, donnant des taux de bonne classification supérieurs à $96 \%$ en utilisant l'ensemble des méthodes abordées et surpassant même les résultats des experts humains. Néanmoins, les particularités de certaines espèces potentiellement plus complexes n'ont pu être résolues et l'élaboration d'un programme informatique qui automatise l'identification des Diatomées, qu'elle que soit leur spécificité, reste encore difficile à résoudre et ne pouvait de ce fait être généralisable. Cependant les recherches continuent et s'appuient fortement sur les retombées de l'Intelligence artificielle. 


\section{REFERENCES}

Afnor NF T90-354, 2007- Qualité de l'eau et détermination de I'Indice Biologique Diatomées (IBD). Décembre, 2007.

Bres S., Jolion J-M., \& Lebourgeois F., 2003Traitement et analyse des images numériques, Hermès éditions,

Combe M., 1975 - Le bassin Rharb-Maamora. Ressources en eau du Plaine du Rharb et bassins du Maroc atlantique. Notes et Mémoires du Service Géologique, Maroc, N ${ }^{\circ}$ 231.

Du Buf H., Bayer M., Droop S., Juggins S., Head R., Fischer S., Bunke H., Wilkinson M., Roerdink J., Cristobàl G., Pech-Pacheco J., Ciobanu A., Shahbazkia H., 1999 - Diatom Identification: a Double Challenge Called ADIAC, In Proceedings ICIAP, pages 734-739.

Deriche R., 2004 - Technique d'extraction de contours, INRIA Sophia-Antipolis.

Dubuisson, 1990. - Diagnostic et reconnaissance des formes, Hermès Paris.

Ébang Menye D., Hubert S., Togouet Z., Foto Menbohan S., Kemka N., Nola M., 2012 Bio-écologie des diatomées épilithiques de la rivière Mfoundi (Yaoundé, Cameroun) : diversité, distribution spatiale et influence des pollutions organiques, 25 (3) : 203-218.

Freeman H., 1961 - On the encoding of arbitrary geometric configurations; IEEE Trans. On Electron Comput. Volume 10, pp 260-268.

Germain H., 1981 - Flore des diatomées, Diatomophycées. Paris, Boubée, 444p.

Guo Q., Chalifour A., Nouboud F., Campeau S., Lavoie I., Mammass D. Et El Yassa M., 2004- Diatom Classification by Image Analysis, IEEE SETIT 2004 International Conference: Sciences of Electronic, Technologies of Information and Telecommunications, Tunisie.

JALBA A.C., Wilkinson M.H.F., Roerdink J.B.T.M., Morphological Hat Transform scale space and their use in pattern classification, Pattern Recognition., 2003 - in The Journal of Pattern Recognition Society.

Kelly M.G., Bayer M.M., H"Urlimann J., Telford R.J., 2002. Human error and quality assurance in diatom analysis. In Automatic Diatom Identification, Series in Machine Perception Artificial Intelligence, chapitre 5, vol.51, World Scientific.
Krammer K. \& Langebertalot H., 1986 Bacillariophyceae. Tome 1: Naviculaceae. Semper Bonis Artibus, 876 p. 1986.

Krammer K. \& Langebertalot H., 1988 Bacillariophyceae. Tome 2: Bacillariaceae, Epithemiaceae et Surirellaceae. Semper Bonis Artibus, $596 \mathrm{p}$.

Krammer K. \& Langebertalot H.1991 Bacillariophyceae. Tome 3: Centrales, Fragilariaceae, Eunotiaceae. Semper Bonis Artibus, $576 \mathrm{p}$.

Krammer K. \& Langebertalot H.1991 Bacillariophyceae. Tome 4: Achnanthaceae. Semper Bonis Artibus, 437p.

Krammer K. \& Langebertalot H. 2000Bacillariophyceae. Tome 5: English and French translation of the Keys. Semper Bonis Artibus, $311 \mathrm{p}$.

Langebertalot H.2001 - Diatoms of Europe -Navicula sensu strict 10 genera separated from Navicula sensulato Frustulia. A.R.G. Gantner Verlag K.G. Ruggell, $526 \mathrm{p}$.

Lavoie I., Hamilton P., Campeau S.2008 - Guide d'identification des diatomées des rivières de l'Est du Canada, 241p.

Loke R.E., Bayer M.M., Mann D.G., Du Bufj.M.H. 2002Diatom recognition by convex and concave contour curvature, Oceans'02 MTS/IEEE.

Michard A. 1976 - Éléments de géologie marocaine. Notes et mémoires du service géologique, Maroc, $\mathrm{N}^{\circ}$ 252, 408P, 221fig. 8tab, 6 pl.

Prygiel J., Coste M.2000 - Guide méthodologique pour la mise en œuvre de l'Indice Biologique Diatomées NF T 90-354, Agences de l'EauCemagref,

Prygiel, J., Carpentier, P., Almeida, S., Coste, M., Druart, J.-C., Ector, L., Guillard, D., Honore, M.A., Iserentant, R., Ledeganck, P., LalanneCassou, C., Lesniak, C., Mercier, I., Moncaut, P., Nazart, M., Nouchet, N., Peres, F., Peeters, V., Rimet, F. Et Rumeau, A. 2002Determination of the biological Diatom. Journal of Applied Phycology, 14: 27-39.

Serra J, 1988- Image Analysis and Mathematical Morphology, volume 2. Academic Press,

Thauvin J.P.1966 - Monographie hydrogéologique de la mamora. Notes et mémoirs du service géologique, Maroc, $\mathrm{N}^{\circ} 195,119 \mathrm{p}$.

Wayne R. 1997- NIH Image to ImageJ: 25 years of image analysis. Nat Methods 9 (7): 671-675. 